\title{
Vorwort
}

\section{Vorwort zur fünfzehnten Auflage}

Die erste Auflage dieses Buches ist vor mehr als 40 Jahren erschienen. Inzwischen hat es mehrere gründliche Überarbeitungen gegeben. Heute darf mit Freude festgestellt werden, dass sich das Buch bewährt hat.

Von jetzt an sind zwei Verfasser verantwortlich. Daniela Lorenz begann ihre akademische Karriere als studentische Hilfskraft bei Lutz Kruschwitz und ist heute Hochschullehrerin an der Julius-Maximilians-Universität Würzburg. Wir haben die letzte in Allein-Autorenschaft verfasste Auflage gründlich durchgesehen und behutsam überarbeitet. Die wichtigsten Änderungen sind folgende:

1. Alle Aufgaben und Musterlösungen wurden aus dem Lehrbuch entfernt, wodurch sich der Umfang des Buches um etwa 100 Seiten verringerte. Neben das Lehrbuch tritt nun ein eigenständiges Arbeitsbuch, dessen Lektüre wir all jenen empfehlen, die Sicherheit im Umgang mit den Methoden der Investitionsrechnung erwerben wollen. Der Fundus der Aufgaben im Arbeitsbuch hat sich gegenüber der früheren (integrierten) Version erhöht.

2. Wir haben zum Lehrbuch Foliensätze im pdf-Format entwickelt, die an Dozenten kostenlos abgegeben werden, wenn diese sich mit einer entsprechenden Bitte an den Verlag wenden. Wer Investitionsrechnung unterrichtet und entsprechenden Bedarf hat, möge sich des Angebots bedienen.

3. Das Kapitel zu Entscheidungen über Investitionsprogramme (unter Sicherheit) ist gegenüber den früheren Auflagen gekürzt worden, wobei wir hoffen, dass nichts Wesentliches verloren gegangen ist.

4. Im Kapitel über Wahlentscheidungen ohne Berücksichtigung von Steuern ist ein Abschnitt hinzugetreten, in dem wir uns mit der Baldwin-Verzinsung auseinandersetzen. Dieses Konzept hat - unter gewissen Voraussetzungen - Vorteile gegenüber dem internen Zinssatz, dem wir im Falle von Wahlentscheidungen nach wie vor kritisch gegenüberstehen.

5. Da der deutsche Steuergesetzgeber seit dem Erscheinen der letzten Auflage wieder einmal nicht untätig war, mussten im Kapitel über Wahlentscheidungen unter Berücksichtigung von Steuern die notwendigen Aktualisierungen vorgenommen werden.

Dass das Literaturverzeichnis dem aktuellen Stand angepasst werden musste, versteht sich von selbst. Bei der Vorbereitung dieser Neuauflage ist uns von Dritten geholfen worden. Besonders zu bedanken haben wir uns bei unserem langjährigen Mitarbeiter T. Ipse.

Berlin und Würzburg, im August 2019

Lutz Kruschwitz und Daniela Lorenz

https://doi.org/10.1515/9783110598025-202 


\section{Vorwort zur vierzehnten Auflage}

Bei jeder Neuauflage des vorliegenden Buches hat mich der Steuergesetzgeber zu Überarbeitungen und Aktualisierungen genötigt. Das ist auch bei der Vorbereitung der 14. Auflage nicht anders gewesen. Da Steuern in diesem Buch eine nicht ganz unbeachtliche Rolle spielen, habe ich mich dazu entschlossen, den entsprechenden Abschnitt aus dem bisherigen zweiten Kapitel herauszulösen und in den Rang eines eigenständigen Kapitels zu erheben.

In den beiden vorangehenden Auflagen hatte ich mich ausführlich mit der Frage beschäftigt, wie bei der Einbeziehung von Steuern in die Investitionsrechnung vorzugehen ist, wenn das Steuersystem - wie zurzeit (nicht nur) bei uns in Deutschland nicht finanzierungsneutral ist. Diesen Abschnitt habe ich jetzt wieder entfernt, weil ich eine angemessene Lösung nur für den ziemlich unrealistischen Fall von einperiodigen Investitionsprojekten präsentieren konnte. Neu aufgenommen wurde ein Abschnitt, in dem investitionsneutrale Steuersysteme beschrieben und analysiert werden.

Des Weiteren wurden die Literaturhinweise auf den neuesten Stand gebracht und kleinere Schwächen beseitigt. Dabei bin ich von Herrn Sebastian Walzel und (wie immer) von Herrn T. Ipse in dankenswerter Weise unterstützt worden.

Lutz Kruschwitz

\section{Vorwort zur dreizehnten Auflage}

Der Steuergesetzgeber hat mich erneut dazu gezwungen, einige Kapitel zu überarbeiten und die erforderlichen Aktualisierungen vorzunehmen. Darüber hinaus habe ich eine grundsätzliche Entscheidung getroffen, die sich auf die statischen Investitionsrechnungen bezieht. Sie sind den dynamischen Verfahren grundsätzlich unterlegen. Früher war es üblich, die statischen Methoden ausführlich zu beschreiben und sie im Anschluss daran kräftig zu kritisieren. Da die Zahl ihrer Verfechter in der Praxis mittlerweile verschwindend klein geworden ist, ist es nicht mehr notwendig, im Detail auf Funktionsweise und Schwächen der statischen Rechnungen einzugehen. ${ }^{1}$ Aus diesem Grunde habe ich den entsprechenden Text auf ein Mindestmaß gekürzt. Die Literatur wurde selbstverständlich auf den neuesten Stand gebracht. Meinem langjährigen Mitarbeiter T. Ipse danke ich wie immer für seine Unterstützung.

1 Siehe dazu Dieter Schneider (2008, S. 605). 


\section{Vorwort zur zehnten Auflage}

Die neue Auflage enthält zwei vollkommen neue Abschnitte: Zum ersten habe ich das Preinreich-Lücke-Theorem aufgegriffen, zum zweiten habe ich mich nach langem Zögern dazu entschlossen, das Thema Realoption zu diskutieren.

Das Preinreich-Lücke-Theorem stellt eine wichtige Grundlage zur Verfügung, wenn man die Zusammenhänge zwischen langfristiger Erfolgsrechnung (Investitionsrechnung) und kurzfristiger Erfolgsrechnung (Kostenrechnung) erörtern will. Das Konzept der Realoptionen übt eine bemerkenswerte Faszination auf viele Leute aus, weil es auf elegante Art und Weise - angeblich - dabei helfen kann, gewisse Schwächen der traditionellen Investitionsrechnung zu überwinden. Ich selbst war immer skeptisch, ob sich die Anhänger des Realoptions-Konzepts tatsächlich auf einem verlässlichen Weg befinden. Nachdem ich aber beobachten musste, dass die Zahl der Anhänger immer weiter stieg, während meine Skepsis vollkommen unverändert blieb, hielt ich es nicht mehr für angebracht, das Thema einfach nur totzuschweigen. Vielmehr beschreibe ich jetzt die Theorie der Bewertung von Finanzoptionen relativ ausführlich und versuche dann, darauf aufbauend zu begründen, warum es ganz und gar unzulässig ist, die Resultate dieser Theorie auf Realoptionen zu übertragen.

Erweitert habe ich den Abschnitt über Kauf und Leasing. Im Zusammenhang mit der Berechnung von kritischen Leasingraten wird jetzt sowohl die Entscheidungssituation des Leasingnehmers als auch die des Leasinggebers betrachtet. Da der Steuergesetzgeber in den letzten beiden Jahren nicht untätig war, mussten Aktualisierungen vorgenommen werden. Selbstverständlich wurde auch die Literatur auf den neuesten Stand gebracht. Aus dem Kreise meiner Mitarbeiter habe ich den Herren Arnd Lodowicks und T. Ipse besonders zu danken. Mein Kollege Ralf Ewert (Johann Wolfgang Goethe-Universität Frankfurt am Main) überließ mir freundlicherweise eine Übungsaufgabe zum Preinreich-Lücke-Theorem.

Lutz Kruschwitz

\section{Vorwort zur siebenten Auflage}

Bei der vorliegenden Neuauflage handelt es sich um einen vollständig überarbeiteten und gestrafften Text. Eine solche gründliche Neubearbeitung erschien zwanzig Jahre nach Erscheinen der Erstauflage dringend erforderlich. Leser, die mit den früheren Auflagen vertraut sind, werden jedoch wissen wollen, was sich neben dem äußeren Erscheinungsbild im Einzelnen geändert hat. Dabei erscheinen mir vor allem drei Sachverhalte erwähnenswert:

1. Der Beurteilung von Investitionsprojekten unter Unsicherheit wurde noch mehr Aufmerksamkeit gewidmet. Im Anschluss an die Theorie der Portfolio-Auswahl 
folgt nun ein vollkommen neuer Abschnitt über die marktorientierte Bewertung riskanter Investitionen.

2. Der Gesetzgeber ist wieder einmal aktiv gewesen und hat das Steuerrecht massiv geändert. Insbesondere sind die Vermögen - und die Gewerbekapitalsteuer weggefallen. Die steuerrechtlichen Darstellungen mussten dieser Entwicklung angepasst werden. Da nun jedoch die für Investitionsentscheidungen wesentlichen Substanzsteuern ganz entfallen sind, konnte ich mich auch im Rahmen des so genannten Standardmodells vollkommen auf die Ertragsteuer konzentrieren.

3. Die früheren Auflagen enthielten Quellcodes für die automatische Durchführung von Berechnungen mit Hilfe von PCs. Diese Programme samt der dazugehörigen Ablaufdiagramme habe ich ersatzlos gestrichen, weil man heutzutage entsprechende Berechnungen zweckmäßigerweise mit Tabellenkalkulationsprogrammen vornimmt.

Die vorliegende Bearbeitung wurde erst möglich, nachdem die alte Auflage mit $\mathrm{LT}_{\mathrm{E}} \mathrm{X} 2 \varepsilon$ neu erfasst worden war. Dabei haben mich insbesondere Frau Renate Mauersberger, Herr Axel Jeromin und Herr T. Ipse tatkräftig unterstützt, wofür ich herzlich danke.

Lutz Kruschwitz

\section{Vorwort zur zweiten Auflage}

Seit dem Erscheinen der ersten Auflage sind sechs Jahre vergangen. Die Grundkonzeption des Buches hat Anerkennung gefunden. Sie blieb daher unverändert. Bei der Neubearbeitung habe ich mich auf das zweite und fünfte Kapitel konzentriert. Dabei wurden folgende Schwerpunkte gesetzt:

1. Die allgemeinen Rechenregeln zur Ermittlung des Endwertes und des Entnahmeniveaus unter beliebigen Kapitalmarktbedingungen wurden vereinfacht. Die Darstellung konnte entsprechend gestrafft werden.

2. Es wurde ein Abschnitt über die Berechnung der Endwerte und Barwerte von Renten eingefügt.

3. Der Abschnitt über das Verfahren der internen Zinsfüße wurde umgearbeitet und neu formuliert. An meiner Ansicht, dass interne Zinsfüße für die Beurteilung von Investitionsalternativen ungeeignet sind, hat sich aber nichts geändert.

4. In einem neuen Abschnitt wird ausführlich auf die Berücksichtigung der Steuern in der Investitionsrechnung eingegangen. Dabei habe ich mich nicht auf Darstellung und Kritik des Standardmodells beschränkt. Vielmehr versuche ich, anhand eines exemplarischen Modellansatzes zu zeigen, wie man Erfolg- und Substanzsteuern detailliert in Investitionsrechnungen einbeziehen kann.

5. Das fünfte Kapitel über Investitionsrechnungen bei unsicheren Erwartungen wurde vollständig überarbeitet. Die Abschnitte über Entscheidungen unter Risiko, se- 
quentielle Investitionsentscheidungen und die Theorie der Portefeuille-Auswahl wurden neu geschrieben.

6. Herkömmliche Lehrbücher über Investitionsrechnung enthalten Auszüge finanzmathematischer Tabellenwerke, deren Benutzung im Zusammenhang mit den dynamischen Investitionsrechnungen empfohlen wird. Meiner Meinung nach sind solche Tabellen beim heutigen Stand der elektronischen Taschenrechner überflüssig geworden. Für künftige Studenten wird der Umgang mit Heim- und Personal-Computern eine Selbstverständlichkeit sein. Deswegen habe ich für wichtige Verfahren der Investitionsrechnung und einige Berechnungsprozeduren im Zusammenhang mit der Portefeuille-Theorie BASIC-Programme angegeben.

Herr Joachim Fischer, Herr Rainer Schöbel und meine Sekretärin, Frau Jutta Breiksch, haben mich in dankenswerter Weise bei der Vorbereitung dieser Auflage unterstützt. Mein Dank gilt ferner allen Lesern, die mich auf Schwächen und Fehler in der ersten Auflage aufmerksam gemacht haben.

Lutz Kruschwitz

\section{Vorwort zur ersten Auflage}

Investitionsrechnungen sind Methoden, mit denen Investitionsentscheidungen auf objektiver Grundlage getroffen werden können.

Die Zahl der Lehrbücher und Fachaufsätze zu diesem Thema ist groß. Für den Anfänger, der damit beginnt, diese Literatur zu studieren, sind insbesondere zwei Dinge verwirrend. Zum ersten zeichnet sich die Theorie der Investitionsrechnung durch eine erhebliche Methodenvielfalt aus. Zum zweiten ist die Qualität einiger Verfahren heftig umstritten. Hinzu kommt die Tatsache, dass einige wichtige Veröffentlichungen auf dem Gebiet der Investitionstheorie in einer recht abstrakten, für den Anfänger nicht immer leicht verständlichen Sprache geschrieben sind.

\section{Zur inhaltlichen Konzeption des Buches}

Das Buch besteht aus drei Teilen:

1. Im ersten Teil werden die Grundlagen der Investitionsrechnung skizziert. Dabei geht es neben den notwendigen Begriffsklärungen vor allem um die Frage, welche Bedeutung die Investitionsrechnungen im Rahmen des gesamten Investitionsentscheidungsprozesses besitzen.

2. Im zweiten Teil werden die wichtigsten Methoden der Investitionsrechnung beschrieben. Dieser Teil ist in drei Kapitel gegliedert, da es drei wichtige Arten von Entscheidungen über Investitionen gibt: 
- Wahlentscheidungen

(Soll das Projekt A oder B oder ... usw. durchgeführt werden?)

- Investitionsdauerentscheidungen

(Soll eine Investition 1 oder 2 oder ... usw. Jahre lang genutzt werden?)

- Programmentscheidungen

(Soll das Investitionsprogramm I oder II oder ... usw. durchgeführt werden?) Alle drei Kapitel beruhen auf der (unrealistischen) Annahme, dass die Unternehmensleitung unter Sicherheit entscheidet, also genau weiß, welche Konsequenzen eintreten werden, wenn sie die eine oder andere Investitionsalternative realisiert.

Um die Vielfalt der Investitionsrechnungen für den Leser überschaubar zu machen und um ihn gleichzeitig in die Lage zu versetzen, die richtige Investitionsrechnung für die richtige Entscheidungssituation auszuwählen, wird immer folgende Vorgehensweise angewandt:

a) Welches Entscheidungsproblem soll gelöst werden?

b) Welches Ziel verfolgt die Unternehmensleitung?

c) Wie sieht die Umwelt der Unternehmung aus?

d) Wie muss man rechnen, um das Entscheidungsproblem in Bezug auf das Entscheidungsziel und unter Berücksichtigung der jeweiligen Umweltsituation $\mathrm{zu}$ lösen (= formale Ableitung der jeweils zweckmäßigen Investitionsrechnungsmethode)?

e) Wie funktioniert die entsprechende Methode, wenn man sie auf ein konkretes Zahlenbeispiel anwendet?

Eines der wichtigsten Ziele dieses Buches ist Folgendes: Der Leser soll lernen, wie man methodisch vorgehen muss, um in einer bestimmten Entscheidungssituation eine angemessene Form der Investitionsrechnung auszuwählen oder selbst zu entwickeln. Man hüte sich davor, irgendwelche Methoden auswendig zu lernen und sie unreflektiert anzuwenden.

3. Im dritten Teil des Buches wird die Annahme aufgehoben, dass Investitionsentscheidungen unter Sicherheit getroffen werden. Damit entsteht die Frage, wie Investitionsrechnungen aussehen müssen, wenn man nicht genau weiß, was geschehen wird, falls man die eine oder andere Investition durchführt. Es geht um das Problem, optimale Investitionsentscheidungen unter Risiko zu treffen. Dabei wird prinzipiell die gleiche Vorgehensweise gewählt wie im zweiten Teil.

In seinen theoretischen Grundlagen beruht das Buch vor allem auf den ausgezeichneten Werken von Hax (1976a) und Dieter Schneider (1975). 


\section{Zur didaktischen Konzeption des Buches}

Jedes Kapitel ist nach folgendem Schema aufgebaut:

1. Am Anfang werden die Lernziele genannt. Lesen Sie die Lernziele bitte aufmerksam durch und prägen Sie sich gut ein, welche Kenntnisse und Fähigkeiten von Ihnen erwartet werden, wenn Sie das Kapitel durchgearbeitet haben.

2. Danach wird der Stoff des jeweiligen Kapitels dargestellt. Wichtige Stellen sind im Text durch Kursivschrift oder Merksätze hervorgehoben. Wenn Sie auf Zahlenbeispiele stoßen, so nehmen Sie bitte Papier und Bleistift zur Hand und versuchen Sie, den Rechengang in allen seinen Einzelheiten genau nachzuvollziehen.

3. Abgeschlossen wird ein Kapitel immer mit gezielten Hinweisen auf ergänzende und vertiefende Literatur.

Die Lektüre dieser Literatur empfiehlt sich dann, wenn Sie die Absicht haben, in den Stoff des Kapitels intensiver einzudringen als dies mit dem vorliegenden Buch möglich ist. Um Ihnen die Auswahl und den Einstieg in das Schrifttum zu erleichtern, habe ich mich auf besonders wichtige Texte beschränkt und in den meisten Fällen kommentierende Bemerkungen angebracht.

Ein ausführliches Literaturverzeichnis finden Sie auf den letzten Seiten dieses Buches. Ergänzend sei auf die umfassenden Quellensammlungen bei Albach (1975) und Lüder (1977) aufmerksam gemacht.

Viele haben dazu beigetragen, dass dieses Buch entstehen konnte: Die inhaltliche Konzeption war Gegenstand von Vorlesungen und Übungen im Fach „Investition und Finanzierung“ an der Technischen Universität Berlin. Durch ihre kritischen Fragen haben mich die Studenten auf viele didaktische, aber auch inhaltliche Mängel und Irrtümer aufmerksam gemacht. Aus dem Kreise meiner Mitarbeiter haben sich die Herren Joachim Fischer und Edgar Stoll die Mühe gemacht, die Manuskripte durchzuarbeiten, $\mathrm{zu}$ kritisieren und zu verbessern. Herrn Fischer verdanke ich viele wertvolle fachliche Ratschläge und Diskussionen sowie das Zahlenbeispiel zur Risikoanalyse. Herr Stoll bewahrte mich aus der Sicht des kritischen Lesers vor zahlreichen Mängeln in der Verständlichkeit des Textes. Die Abbildungen hat Herr Gernot Meißner gezeichnet, und Frau Marianne Ludwig bewies erstaunliche Geduld und großes Einfühlungsvermögen beim Schreiben meiner meist nur schwer lesbaren Manuskripte. 
